\title{
273.
}

\section{NOTE SUR LA TRANSFORMATION DE TSCHIRNHAUSEN.}

[From the Journal für die reine und angewandte Mathematik (Crelle), tom. LVIII. (1861), pp. $259-262$.

ON trouve dans le mémoire de $\mathrm{M}$. Hermite "Sur quelques théorèmes d'algèbre et la résolution de l'équation du quatrième degré" (Comptes Rendus, t. xLvi. p. 961, 1858) un théorème très-important relatif à la transformation de Tschirnhausen, à l'aide de laquelle une équation $f(x)=0$ est transformée en une autre du même degré en $y$ par la substitution $y=\phi x$, où $\phi x$ désigne une fonction rationnelle. En considérant pour fixer les idées une équation du quatrième degré

$$
\left(a, b, c, d, e^{\gamma} x, 1\right)^{4}=0,
$$

M. Hermite donne à l'équation $y=\phi x$ la forme que voici,

$$
y=a T+(a x+4 b) T_{0}+\left(a x^{2}+4 b x+6 c\right) T_{1}+\left(a x^{3}+4 b x^{2}+6 c x+4 d\right) T_{2},
$$

et il fait voir que les coefficients de la transformée en $y$ ont la propriété suivante: toute fonction de ces coefficients, laquelle exprimée en fonction de $a, b, c, d, e, T, T_{0}, T_{1}, T_{2}$ ne contient pas $T$, est un invariant, c. à d. un invariant des deux fonctions

$$
(a, b, c, d, e \gamma \xi, \eta)^{4}, \quad\left(T_{0}, T_{1}, T_{2} \gamma \eta,-\xi\right)^{2} .
$$

Cela revient à dire qu'en supposant la valeur de $T$ déterminée de manière à faire évanouir dans l'équation en $y$ le coefficient du second terme (de $y^{3}$ ), les autres coefficients seront des invariants, de sorte que, si dans le polynome en $y$ qui est égalé à zéro on considère $y$ comme une constante absolue, le polynome tout entier sera un invariant des deux fonctions ci-dessus mentionnées. On trouve sans peine la valeur que doit avoir $T$, elle est donnée par l'équation

$$
0=a T+3 b T_{0}+3 c T_{1}+d T_{2},
$$


ce qui conduit pour $y$ à la valeur

$$
y=(a x+3) T_{0}+\left(a x^{2}+4 b x+3 c\right) T_{1}+\left(a x^{3}+4 b x^{2}+6 c x+3 d\right) T_{2},
$$

et en même temps la transformée en $y$ aura la propriété dont il s'agit.

Par rapport à la forme de l'expression que l'on vient de trouver pour $y$ il est bon de remarquer que les coefficients numériques qu'on y rencontre, hormis ceux du terme en $x^{0}$, ou de $b T_{0}+3 c T_{1}+3 d T_{2}$, sont les coefficients de la puissance $(1,1)^{4}$, tandis que les coefficients qui ont été désignés comme faisant exception sont ceux de la puissance $(1,1)^{3}$. Une remarque pareille s'applique au cas général. Pour démontrer le théorème énoncé, je représente l'équation qui vient d'être écrite par $y=V$, la transformée en $y$ sera donc

$$
\left(y-V_{1}\right)\left(y-V_{2}\right)\left(y-V_{3}\right)\left(y-V_{4}\right)=0,
$$

où $V_{1}, V_{2}, V_{3}, V_{4}$ sont ce que devient $V$ lorsqu'on y substitue successivement pour $x$ les quatre racines de l'équation $\left(a, b, c, d, e \gamma(x, 1)^{4}=0\right.$. Or, en considérant $y$ comme une constante, pour que l'expression qui forme la première partie de l'équation que l'on vient d'écrire soit un invariant, les conditions à remplir consistent en ce que cette expression se réduise à zéro au moyen de l'un et l'autre des opérateurs

$$
\begin{array}{r}
a \partial_{b}+2 b \partial_{c}+3 c \partial_{d}+4 d \partial_{e}-\left(T_{2} \partial_{T_{1}}+2 T_{1} \partial_{T_{0}}\right), \\
4 b \partial_{a}+3 c \partial_{b}+2 d \partial_{c}+e \partial_{d}-\left(2 T_{1} \partial_{T_{2}}+T_{0} \partial_{T_{1}}\right) .
\end{array}
$$

Ces - conditions seront satisfaites si chacun des facteurs $y-V_{1}$, etc. est doué de cette même propriété, c. à d. si $y-V$, ou plus simplement si $V$, en y faisant $x$ égal à l'une des racines de l'équation en $x$, se réduit à zéro au moyen de l'un et l'autre des deux opérateurs ci-dessus écrits. Je considère le premier des deux opérateurs et pour abréger je le désigne par

$$
\Delta-\left(T_{2} \partial_{T_{1}}+2 T_{1} \partial_{T_{0}}\right)
$$

Pour avoir $\Delta V$, il faut d'abord former la valeur de $\Delta x$. On l'obtient en opérant avec $\Delta$ sur l'équation $(a, b, c, d, e \chi x, 1)^{4}=0$, ce qui donne

$$
\left(a, b, c, d \gamma(x, 1)^{3} \Delta x+(a, b, c, d \gamma x, 1)^{3}=0 \text {, ou } \Delta x=-1 .\right.
$$

La partie de $\Delta V$ qui dépend de la variation de $x$ est par conséquent

$$
-a T_{0}+(-2 a x-4 b) T_{1}+\left(-3 a x^{2}-8 b x-6 c\right) T_{2} .
$$

Pour l'autre partie de $\Delta V$ on trouve aisément

$$
a T_{0}+(4 a x+6 b) T_{1}+\left(4 a x^{2}+12 b x+9 c\right) T_{2},
$$

et de là en ajoutant

$$
\Delta V=2(a x+b) T_{1}+\left(a x^{2}+4 b x+3 c\right) T_{2}
$$

ce qui est précisément égal à

$$
\left(T_{2} \partial_{T_{1}}+2 T_{1} \partial_{T_{0}}\right) V
$$


Done $V$ se réduit à zéro par l'opérateur

$$
\Delta-\left(T_{2} \partial_{T_{1}}+2 T_{1} \partial_{T_{\mathrm{o}}}\right)
$$

De même en représentant le second opérateur par

on trouve d'abord

$$
\nabla-\left(2 T_{1} \partial_{T_{2}}+T_{0} \partial_{T_{1}}\right)
$$

$$
\left(a, b, c, d \gamma(x, 1)^{3} \nabla x+x \cdot\left(b, c, d, e^{\gamma}(x, 1)^{3}=0,\right.\right.
$$

mais en ayant égard à l'équation $\left(a, b, c, d, e \gamma(x, 1)^{4}=0\right.$ la valeur de $\nabla x$ se réduit à $\Delta x=x^{2}$. La partie de $\nabla V$ due à la variation de $x$ est par conséquent

$$
a x^{2} T_{0}+\left(2 a x^{3}+4 b x^{2}\right) T_{1}+\left(3 a x^{4}+8 b x^{3}+6 c x^{2}\right) T_{2} .
$$

L'autre partie de $\nabla V$ est

$$
(4 b x+3 c) T_{0}+\left(4 b x^{2}+12 c x+6 d\right) T_{1}+\left(4 b x^{3}+12 c x^{2}+12 d x+3 c\right) T_{2} .
$$

En les ajoutant, le coefficient de $T_{2}$ s'évanouit en vertu de l'équation en $x$, et l'on trouve

$$
\nabla V=\left(a x^{2}+4 b x+3 c\right) T_{0}+2\left(a x^{3}+4 b x^{2}+6 c x+3 d\right) T_{1},
$$

ce qui est précisément égal à

$$
\left(2 T_{1} \partial T_{T_{2}}+T_{0} \partial_{T_{1}}\right) V
$$

$V$ se réduit donc à zéro au moyen de l'opérateur

$$
\nabla-\left(2 T_{1} \partial_{T_{2}}+T_{0} \partial_{T_{1}}\right),
$$

ce qui achève la démonstration dont il s'agissait. Il va sans dire que la démonstration serait conduite d'une manière semblable pour une équation de degré quelconque. Pour avoir l'exemple le plus simple, je prends les équations

$$
\begin{gathered}
\left(a, b, c, d \gamma(x, 1)^{3}=0,\right. \\
y=(a x+b) T_{0}+\left(a x^{2}+3 b x+2 c\right) T_{1},
\end{gathered}
$$

et pour effectuer l'élimination j'écris

$$
\begin{aligned}
& y x=\left(a x^{2}+b x\right) T_{0}+(-c x-d) T_{1}, \\
& y x^{2}=\left(-2 b x^{2}-3 c x-d\right) T_{0}+\left(-c x^{2}-d x\right) T_{1} .
\end{aligned}
$$

Maintenant on a les trois équations

$$
\begin{aligned}
& 0=b T_{0}+2 c T_{1}-y+x\left(a T_{0}+3 b T_{1}\right)+x^{2} \cdot a T_{1}, \\
& 0=-d T_{1}+x\left(b T_{0}-c T_{1}-y\right)+x^{2} \cdot a T_{0}, \\
& 0=-d T_{0}+x\left(-3 c T_{0}-d T_{1}\right)+x^{2}\left(-2 b T_{0}-c T_{1}-y\right)
\end{aligned}
$$

donc l'équation en $y$ est

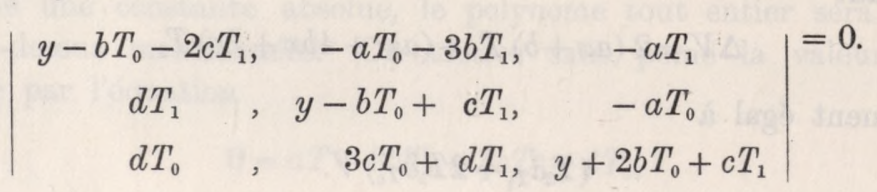


En ordonnant ce déterminant suivant les puissances de $y$, les coefficients de $y^{3}, y^{2}, y, y^{0}$ deviennent des formes binaires en $T_{0}, T_{1}$, des ordres $0,1,2,3$. En calculant les valeurs de ces quatre coefficients on les trouve respectivement

$$
\begin{aligned}
& =1, \\
& =0 \\
& =3\left(a c-b^{2}, a d-b c, b d-c^{2} \gamma T_{0}, T_{1}\right)^{2}, \\
& =\left(a^{2} d-3 a b c+2 b^{3}, 3 a b d-6 a c^{2}+3 b^{2} c,-3 a c d+6 b^{2} d-3 b c^{2},-a d^{2}+3 b c d-2 c^{3} \gamma\left(T_{0}, T_{1}\right)^{3},\right.
\end{aligned}
$$

c'est-à-dire que l'équation en $y$ est celle-ci :

$$
y^{3}+3 y\left(a c-b^{2}, a d-b c, b d-c^{2} \gamma T_{0}, T_{1}\right)^{2}
$$

$$
+\left(a^{2} d-3 a b c+2 b^{3}, 3 a b d-6 a c^{2}+3 b^{2} c,-3 a c d+6 b^{2} d-3 b c^{2},-a d^{2}+3 b c d-2 c^{3} \gamma T_{0}, T_{1}\right)^{3}=0,
$$

équation qui remplit en effet la condition ci-dessus mentionnée, d'avoir pour coefficients des invariants des deux formes:

$$
(a, b, c, d \gamma \xi, \eta)^{3}, \quad\left(T_{0}, T_{1} \chi \eta,-\xi\right) .
$$

Dans le cas particulier dont il s'agit, la fonction $\left(T_{0}, T_{1} \chi_{\eta} \eta-\xi\right)$ est linéaire, et l'on peut même dire que les coefficients sont des covariants de la seule fonction $\left(a, b, c, d \gamma T_{0}, T_{1}\right)^{3}$ en y considérant $T_{0}, T_{1}$ comme les variables.

J'ai cru qu'il y avait de l'intérêt de donner cette vérification. D'ailleurs je remarque qu'au moyen du théorème même on aurait pu trouver tout de suite l'équation en $y$, en écrivant d'abord $T_{1}=0$, ce qui donne le système

$$
\begin{gathered}
\left(a, b, c, d \gamma(x, 1)^{3}=0,\right. \\
y=(a x+b) T_{0},
\end{gathered}
$$

et de là

$$
\frac{1}{a}\left(a, b, c, d \gamma y-b T_{0}, a T_{0}\right)^{3}=0
$$

ou enfin

$$
y^{3}+3 y\left(b^{2}-a c\right) T_{0}^{2}+\left(a^{2} d-3 a b c+2 b^{3}\right) T_{1}^{3}=0 .
$$

Les valeurs des coefficients peuvent être complétées, eu égard à ce qu'ils doivent être des invariants de $(a, b, c, d \gamma \xi, \eta)^{3},\left(T_{0}, T_{1} \chi \eta,-\xi\right)$ (ou, comme on vient de le dire, des covariants de $\left.\left(a, b, c, d \gamma T_{0}, T_{1}^{\prime}\right)^{3}\right)$. Mais ce n'est que dans le cas particulier, où les coefficients $T_{0}, T_{1}$ sont au nombre de deux, que l'on peut réduire la seconde équation à une équation linéaire.

Londres, 18 Avril 1860. 